REgULATION POSTTRANGCRIPTIONNELLE DE L'EXPRESSION DU GENE DE LA GLYCEROPHOSPHATE DESIIYDROGENASE PAR LA DEXAMETHASONE DANS L ADIPOCYTE 3T3-E412A.

Mousitaid N. L, Hainque B. 1, Juignard-lboulange A. l, Pairault. J.2

I.N.S.I.R.M. H.177, Paris, 21.N.S.I.R.M., I.282 Créleil, France.

Nous avons précédemment montré que la dexamethasone (DEX) inhibe l'activite glycerophosphate deshydrogenase (GPDH) au cours de la differenciation terminale des préadipocytes $3 \mathrm{~T} 3-\mathrm{F} 442 \mathrm{~A}$. Dans ce travail, nous avons voulu savoir si la DEX exerce le méme effet sur des cellules differenciés et par quels mécanismes.

L activite enzymatigue ainsi gue le taux de son messager sont nesurés apres 24 ou $48 \mathrm{~h}$ de traitement par la DEX (2OnM) en presence ou en absence d'insuline (IOn!1). La demi-vie du messager est estimee apres inhibition de la synthese des ARNm par le 5,6 dichloro 1-B-D ribofluoranosyl benzimidazole (DRB) et analyse des ARN totaux selon la technique du northern blot a l'aide de différentes sondes cDNA (GPDH, actine et adipsine).

En absence d'insuline, l'activité GPDH n'est diminuée de 50q qu'apres $48 \mathrm{~h}$ de traitement par la DEX aloss que $24 \mathrm{~h}$ suffisent pour reduire d'un meme facteur la guantité de l'ARNm. La demi-vie du messager est egalement diminuée par la $\mathrm{DEX}$ ( $5 \mathrm{~h}$ vs $8 \mathrm{~h}$ pour le temoln). Par contre, la DEX régule de façon positive l'expression du gene de l'actine et ne modifie pas celle de 1 adipsine. La presence d'insuline s'oppose aux effets de la DEX et en particulier stabilise le messager de la GPDH $(\mathrm{T} 1 / 2>20 \mathrm{~h})$.

Ces résultats montrent que la DEX régule a un niveau posttranscriptionnel l'expression du gene de la GPDH dans l'adipocyte 3T3-F442A et suggerent un effet specifique de la DEX sur l'expression de ce messager.

\title{
CONTROLE TRANSCRIPTIONNEL DES ACTIVITES STEROIDOGENES DU CORTEX SURRENAL PAR LE FACTEUR DE TRANSFORMATION TGF-B
}

Feige J.J., Cuchet C., Pascal 0., Defaye G., Perrin A., Rainey W., Madani C., Chambaz E.M. BRCE, INSERM U ?44, LBIO, DRF, Centre d'Etudes Nucléaires, $85 x, 38041$ Grenode Cedex

Les TGF- $B$ (transforming growth factors $B$ ) constituent une famille de peptides ubiquitaires et doucs d'activités plēitropiques. Selon les cellules cibles, ils sont des stimulateurs (certains fibroblastes) ou des inhibiteurs (cellules épithéliales) de croissance ou des régulateurs de la différenciation (cellules êpithéliales bronchiques, préadipocytes).

Notre groupe s'est at taché à caractériser les effets de TGF- $\beta$ sur les ceilules corticosurrénales bovines en culture primaire. TGF- $\beta$ n'est pas mitogène pour ces cellules et ne modifie pas la croissance induite par le fibroblast growth factor basique (b-FGF). Par contre, TGF-B inhibe très efficacement $\left(I_{50}=0,2-0,4 \mathrm{ng} / \mathrm{ml}\right)$ la production de corticostêroides, aussi bien basale qu'induite par I'ACTH ou 1 ângiotensine II. La demi inhibition est observẻe après 6 heures de traitement par TGF-B (1 na/m1) et l'inhibition maximale est obtenue après 12-15 heures d'exposition. TGF- $B$ exerce cet effet en agissant similtanément sur plusieurs cibles : diminution du nombre de récepteurs de l'anciotensine II, perte d'activité stéroide $17 \alpha$-hydroxylase (cytochrome P-450, $17 \alpha$ ), enzyme clef dans la voie de biosynthèse des corticostêroìdes. A l'aide d'un anticorps et d'une sonde ADNc spécifiques de la $17 \alpha$-hydroxylase, nous avons pu montrer que la cinétique de la diminution d'activité se superpose a celle de la disparition de la protéine enzymatique et est précédée par une diminution de l'ARNm correspondant (50 \% de diminution de l'ARNm aprēs 3-4 heures de traitement par TGF-B). De plus, TGF-B bloque l'auginentation de l'ARNm, de la protēine et de l'activité $17 \alpha$-hydroxylase induite par l'hormone majeure de diffórenciation surrēnale, 1 'ACTH.

Nous avons êgalement mis en évidence, dans les cellules corticosurrênales, l'existence de récepteurs à TGF-B, dont le nombre apparait régulé par T'ACTH. La démonstration récente d'une synthèse active de TGF-B par ces cellules nous permet de proposer ce facteur comme un régulateur autocrine des activités différenciēes du cortex surrénal. 